\title{
A Quark-Independent Description of Confinement
}

\author{
Nicolae Mazilu
}

\begin{abstract}
Currently the quantitative description of confinement inside nuclear matter is exclusively limited to computer experiments, mainly on lattices, and concentrating upon calculating the static potential. There is no independent reference for comparison and support of the results, especially when it comes to the quark potential in the continuum limit. Yet, we are entitled to be optimistic, for the basic results of these calculations seem to be correct from an entirely different point of view, suggested by Manton's geometrization of Skyrme theory. The present work shows the reasons of this point of view, and offers a static potential that might serve as independent reference for comparison and endorsement of any lattice calculations, and in fact of any structural hypotheses of nuclear matter. A historical review of the pertinent key moments in the history of modeling of nuclear matter, as well as an outlook anticipating the necessary future work, close the argument.
\end{abstract}

Key Words: Nuclear Matter, Static Quark Potential, Manton's Geometrization, Skyrmions, Confinement, Temperature, Sufficient Statistic, Exponential Distributions, Parameter of a Distribution, Quarks, Partons, Rational Maps

\section{Introduction}

Nuclear matter is a concept on whose explanation one heavily relies upon analogies with classical achievements in the phenomenology of matter at large: solids, liquids, gases, plasmas, and their interrelationship (Satz, 2011). There are, nevertheless, exquisite achievements, driven by characteristic experiments, uniquely belonging to the field of nuclear matter. These could add in understanding the concept of matter in general, in that they could help improve the very classical phenomenological models serving as starting points of analogies. Yet, the theoretical physics rarely, if ever, backtracks its own concepts, in order to improve them based on the gain of knowledge occasioned by the analogies to which these concepts serve as starting points. In some cases however, such a retracing becomes a critical need. This work is addressed to such a case.

A place where the theoretical physics turned to a special classical description of matter is the geometry related to the theory of Skyrme. One can hardly deny, indeed, the classical source of inspiration of the exquisite work of Nicholas Manton on the geometrization of skyrmions (Manton, 1987). As indicated by the author himself, it is simply the theory of large deformations. The deformation of matter is definitely its most general property, expected therefore to be effectual everywhere, even in nuclear matter. Getting into specific details, however, one can say that Manton has chosen for analogy only a particular model of the deformation of rubbers - of polymers, in general - the so-called Mooney-Rivlin model for the density of energy of deformation (for an even more detailed discussion of this point see Mazilu, Agop, 2012). What we intend to do here, is to extend first the very classical source of inspiration of Manton's geometrization, by emphasizing the fact that the theory of large deformations itself can be considered as a kind of gauge theory. This quality is likewise passed unto nuclear matter's description, whereby the skyrmions are the explicit expression of its space expanse. Then we indicate the gauge freedom and its relationship with the classical theory of deformation. Based on this, and on the idea that every stage of deformation requires a 
kind of equilibrium of the structured matter, one can then offer a physical reason, and in fact a reasonable explanation, for the so-called rational maps. This, in turn, leads to an expression of the confining potential of nuclear matter, independent of its structure, which can be taken for reference in guiding the researches on the functional form of the static quark potential, or any other kind of static nuclear potential for that matter (see Bali, 2001, for the details regarding this topic, and also Alkofer, Greensite, 2007 for a relevant review of the problem).

One cannot deny either the great advantage of rational maps in understanding the structure of the nuclear matter, but the point of the present work is that one can say a lot more about them by the way of principle. In this respect, the Manton's geometrization of skyrmions is instrumental. Indeed, the Skyrme variational energetic principle leads to skyrmions as solutions of a nonlinear differential equation. In the early sixties of the last century it was noticed that while such equations can well represent the space extension of physical particles, and thus may help withdraw these concepts from the highly artificial classical condition of material points (Enz, 1963, 1964, 2006), some of them might have unstable solutions. This occurrence is in disagreement with the precepts of physics of particles (Hobart, 1963). The main variational property of the static solutions of nonlinear equations, taken into consideration as reference in defining the extension of particles, was their stability against so-called uniform scaling of the coordinates (Derrick, 1964). This is indeed the main property that differentiates an extended particle model from its classical counterpart, which is simply a point characterized, besides its coordinates of position, by qualities like mass, charge, color, etc. With Manton's geometrization one can go beyond the uniform scaling, and indeed into the deformation of nuclear matter, thus taking in consideration even the anisotropy of particles (Manton, 2009). It is here the place where the rational maps can prove their true virtues, at least from numerical calculations' point of view (Charalampidis, Ioannidou, Manton, 2010; Manton, 2012). While currently these maps are just working hypotheses, it seems however that they should have a rational basis, at least when considered as part of the acquisition of knowledge. As shown in the present work, they turn out to be relations between the averages over the ensembles of equilibrium, and the parameters of their distributions functions, as long as these distributions are of the classical exponential type. Related to this conclusion, we would like to advance a certain point of view, mostly related to post-Newtonian natural philosophy, in the particular problem of the structure of nuclear matter. The general philosophical reasons of this point of view rest upon the idea of confinement of matter which, physically, is in fact closer to the continuum approach of the deformation of matter than to the idea of particulate forces usually taken into consideration. In this respect, the Manton's geometrization appears to be the peak realization of theoretical physics when it comes to theoretical approach of nuclear matter, for we are hardly aware of its internal structure. What we can certainly grasp in specific experiments is that this matter is in a permanent deformation. It is this process that needs to be properly described, and we suggest here a physical basis for such a description.

\section{Resumé of Experimental Approach to the Theory of Large Deformations}

The main idea is that the materials' behavior under stretching is a consequence of the interior forces acting between their constitutive particles - classically, the molecules. So, by performing loading experiments of material specimens, we actually get insight into the structure of the materials. The primary experimental recordings are stretches, i.e. the relative changes of the dimensions of specimens of material under load, and the loading forces, which can then be recorded as a function of these stretches. A typical 
uniaxial loading curve for a cured rubber, i.e. a curve obtained with the loading force acting along a given direction, is shown in Fig. 1. The data is represented as the nominal stress $\sigma$, vs. nominal strain, $\varepsilon$. The nominal stress is defined as the force divided by the initial cross-sectional area of the specimen, and the nominal strain is the variation of length of the specimen along the direction of loading, divided by its initial length on that direction.

Such a curve is used in applications of constitutive modeling, as follows: the work of loading force, invested in the deformation of the specimen, is calculated in the usual way, as the area under the loading curve. In terms of stretch, this comes to the integral

$$
\mathrm{U}\left(\lambda, \lambda_{0}\right)=\int_{\lambda_{0}}^{\lambda} \sigma(\mathrm{x}) \cdot \mathrm{dx} \quad \therefore \quad \sigma(\lambda)=\frac{\mathrm{d}}{\mathrm{d} \lambda} \mathrm{U}\left(\lambda, \lambda_{0}\right)
$$

Here $\lambda$ denotes the stretch, the suffix zero meaning an initial already stretched state, if the case may occur. Therefore, if one finds the function $\mathrm{U}\left(\lambda, \lambda_{0}\right)$, one is able to calculate the nominal stress. In fairly natural assumptions this allows then to perform calculations regarding the behavior of the material under stress in general conditions, viz. to model the behavior of the material.

The fundamental assumption implicitly used in any such constitutive modeling is that the shape of the loading curve is uniquely defined by the material, and that it reflects the specific forces between the structural constituents of the material. Therefore, for a three-dimensional specimen, the work spent in the loading reflects the energy of those internal forces, and is a priori considered as a potential for the stress along the directions of stretches:

$$
\mathrm{W}=\mathrm{U}\left(\lambda_{1}, \lambda_{2}, \lambda_{3}\right) \quad \therefore \quad \delta \mathrm{W} \equiv \sum \frac{\partial \mathrm{U}\left(\lambda_{1}, \lambda_{2}, \lambda_{3}\right)}{\partial \lambda_{\mathrm{k}}} \delta \lambda_{\mathrm{k}}=\sum \mathrm{f}^{\mathrm{k}}\left(\lambda_{1}, \lambda_{2}, \lambda_{3}\right) \delta \lambda_{\mathrm{k}}
$$

This assumption comes down to the idea that $\mathrm{f}^{\mathrm{k}}$ are the principal components of the stresses generated by the internal forces inside the material. At least in the case where the stress is a symmetric matrix this works very well, with outstanding results. If, on the other hand, it is assumed further that the stress is a tensor with respect to the rotation group of euclidean space, the equation above can be understood as giving a tensorial invariant - the trace of the product between the tensor of stress and that of strain.

Now, another assumption comes usually into play, namely that the stretches actually define a coordinate transformation in the space occupied by matter. In this case the strain tensor can be taken as quadratic in stretches, so that the nominal strain is just a singular case of definition of the measure of strain. Expressed as a function of strain only, the potential $U$ must be invariant, therefore a function of the invariants of strain tensor. These are

$$
\mathrm{I}_{1}=\lambda_{1}^{2}+\lambda_{2}^{2}+\lambda_{3}^{2} ; \quad \mathrm{I}_{2}=\lambda_{2}^{2} \lambda_{3}^{2}+\lambda_{3}^{2} \lambda_{1}^{2}+\lambda_{1}^{2} \lambda_{2}^{2} ; \quad \mathrm{I}_{3}=\lambda_{1}^{2} \lambda_{2}^{2} \lambda_{3}^{2}
$$

A linear combination of the first two of these invariants is used by Nicholas Manton in his analogy, and amounts to the so-called Mooney-Rivlin model of large deformation (Mooney, 1940; Rivlin, 1948)

However, the most general potential should be obtained by curve-fitting the loading curve, and then integrating this result, as indicated in equation (1). Such a potential is expected to represent the properties of the confining forces that keep the material together. In view of the Manton's idea of geometrization, the things should go the very same way for the nuclear matter. Therefore, at least qualitatively speaking, what 
we obtain for rubbers should go equally for nuclear matter: the functional form of the static potential should be the same. At least for the case of 'uniform scaling' this seems to be indeed the case. Once we have thus obtained a reliable functional form of potential, the hard part of the problem, i.e. the physical explanation of such a potential, can be handled on a sound basis. The current usual theoretical approach though, is by constructing a partition function based upon a priori assumptions regarding the structure of the material. This approach is closely followed even in nuclear calculations. In broad lines we also will follow it here. The only difference is that we will be concerned not with the partition function, but directly with the statistical distribution function itself. This method is intended to help avoiding the concept of temperature as a primary parameter of the equilibrium distributions, replacing it with a gauge phase, as it seems only natural when experiments are considered in detail. The temperature is in general a classical remnant of a sufficient statistic, which works properly only in the highly idealized case of a classical gas. The nuclear matter is, nevertheless, far away from this condition.

\section{A Statistical Theory of Experimental Loading Curves}

The experimental data for the uniaxial loading of rubbers (and not only rubbers!), can be aptly represented by a formula like

$$
\sigma=\mathrm{a}+\mathrm{b} \cdot \tan \varphi
$$

Here $\varphi$ is a phase angle depending on the evolution of deformation. At reasonable values of deformation this angle can be taken as a linear function of a measure of deformation. Such a choice is represented in Fig. 2. One can see that the choce might not be appropriate all along the curve, but we take it as it is, just to built our argument, which is based on the idea that the phase angle depends somehow on the stage of deformation.

Fact is that one can find many other, perhaps more convenient, functions to fit the experimental curve from Fig. 1, as is in fact the case in the practice of constitutive modeling. The Fig. 3 shows also a curve fit of the data but this time with a rational function - a ratio of two polynomials, one of third degree, the other of second degree in the nominal strain. It is not unusual, when it comes to representing the experimental data, to approximate the tangent function by a rational one, but the occasion seems to call for a deeper reason for the matching of the two curve fits. As we mentioned, one can see that the tangent fit is not quite as good as the rational one in the first place, and so one can be induced into thinking that it refers to an ideal situation which, obviously, cannot be reached in the real experiments. A comparison of Figs. 2 and 3 seems to demonstrate that in reality the data is better represented by the rational function, which thus should carry an important physical meaning. The point is, however, that the two are not independent: the rational fit from Fig. 3 is actually inferred by starting from equation (4). Let's describe the procedure.

Based on the third principle of classical mechanics, one can intuitively say that the right hand side of equation (4) must be a kind of mean over the ensemble of equilibrium forces between the molecules of rubber. The formula (4) then reflects only partially the structure of this mean, while showing something more: in view of the good representation from Fig. 2, one can infer that the parameters of this equilibrium depend on the stage of deformation process, reflected by the measure of this deformation, entering the argument of the tangent function from equation (4). The problem would then be only to find a suitable statistics, in order to be able to characterize this mechanical equilibrium as a statistical one. This appears to be a proper approach, for one cannot know a priori if the internal forces between molecules are acting 
independently of each other, in order to apply, for instance, the principle of linear independence, and thus to consider the load as balanced by a resultant of molecular forces. So much the more, we cannot say anything a priori about the forces internal to nucleus, for that matter.

Now, the formula (4) has the typical form of an eigenvalue of the stress matrix in what we would like to call the Barbilian representation (Mazilu, 2006; Mazilu, Agop, 2012) whereby the argument of the function (4) is the arbitrary phase of the local shear vector in an octahedral plane, and is represented by a gauge angle $\phi$. If, as usually in the experimental practice, we accept such an identification for the phase $\varphi$ from equation (4), then we also have to accept the apparently natural idea that the stage of deformation is controlled by the octahedral shearing of the material, i.e. by a measure of motions of the material out of the line of action of external force. This conclusion is, again, coping both with our intuitive image of deformation process, and with the definition of stresses as equilibrium quantities. If the stress in the form given in equation (4) is then interpreted as an average over the fluctuations of the internal forces between the molecules, as reflected in the Barbilian phase values, then the statistical nature of the ensemble undergoing this equilibrium, manifested in the distribution function characterizing it, is easy to obtain. In order to show this, we simply differentiate equation (4) with respect to $\phi$ and then eliminate this parameter in the resulting expression. The result is, in general, a differential equation of Riccati type:

$$
\frac{\mathrm{d} \sigma}{\mathrm{d} \phi}=\mathrm{A} \sigma^{2}+2 \mathrm{~B} \sigma+\mathrm{C}
$$

where A, B, C are some constants.

In order to get a further insight into the nature of the statistics we are after, let's notice that the most general noncommittal probability distribution that one can obtain from experimental data, having at disposal only the values of the mean is, according to the principle of maximum information entropy for instance, an exponential distribution. If $\mathrm{X}$ is the physical quantity to be statistically characterized, and $\theta$ is either a measured value, or a certain function of the measured value, then the exponential distribution of the values $\xi$ of $\mathrm{X}$, over a certain ensemble of allowed values, is given by

$$
P_{\theta}(x)=\theta \cdot e^{-\theta \xi}
$$

Now, in real cases it may happen that $\mathrm{X}$ is not allowed to run over the whole real positive axis. This may be due to the fact that the real axis representing the values of this quantity is already endowed with an a priori measure, or to the fact that $\mathrm{X}$ has indeed a limited range - i.e. there is a partition of real axis representing $\mathrm{X}$ - which, from mathematical point of view, comes down to the same model. The model then produces the following family of elementary probabilities depending on one experimental parameter $\theta$ :

$$
\mathrm{F}_{\mu}(\mathrm{d} \xi \mid \theta)=\left[\mathrm{N}_{\mu}(\theta)\right]^{-1} \mathrm{e}^{-\theta \xi} \mu(\mathrm{d} \xi)
$$

Here $\mu(\mathrm{d} \xi)$ is an a priori measure of the real line, and the normalization factor $\mathrm{N}_{\mu}$ is defined by equation

$$
\mathrm{N}_{\mu}(\theta) \equiv \int_{-\infty}^{\infty} \mathrm{e}^{-\theta \xi} \mu(\mathrm{d} \xi)
$$


These exponential probability distributions, used currently in all kinds of physical and engineering applications, have the remarkable property, independent of the a priori measure $\mu(d \xi)$ of the real numbers, that their variance (VAR) can be related to their mean $(\mathrm{m})$ by

$$
\operatorname{VAR}(\xi)=\frac{\mathrm{dm}(\theta)}{\mathrm{d} \theta} ; \quad \mathrm{m}(\theta) \equiv \int_{\text {Range }} \xi \mathrm{dF}_{\mu}(\xi \mid \theta) ; \quad \operatorname{VAR}(\xi) \equiv \int_{\text {Range }} \xi^{2} \mathrm{dF}_{\mu}(\xi \mid \theta)-[\mathrm{m}(\theta)]^{2}
$$

A particular subclass of exponentials, with large applications in experiments as well as in theory, is the family of distributions with quadratic variance function. For these, the variance, considered as a function of mean, is a quadratic polynomial. Their mean satisfies therefore the Riccati differential equation

$$
\frac{\mathrm{dm}(\theta)}{\mathrm{d} \theta}=\mathrm{r}_{1} \mathrm{~m}^{2}+2 \mathrm{r}_{2} \mathrm{~m}+\mathrm{r}_{3}
$$

where $r_{1}, r_{2}, r_{3}$ are three real constants characterizing the distribution, and accessible to measurement.

Comparing our differential equation (5) for experimental stresses with equation (10) for the mean of a quadratic variance distribution function, one can say that the experimental stress is the mean over an ensemble of forces characterized by a quadratic variance distribution. Provided, of course, we interpret the phase angle $\phi$ as the parameter of a family of such distributions characterizing our large deformations. In other words, the parameter of this ensemble - the analogous of temperature from the case of classical ideal gas - characterizes the capability of extended matter to deflect the action of the external force from the direction of its action. Under the condition $r_{1} r_{3}-r_{2}^{2}>0$

$$
\mathrm{m}(\theta)=-\frac{\mathrm{r}_{2}}{\mathrm{r}_{1}}+\frac{\sqrt{\mathrm{r}_{1} \mathrm{r}_{3}-\mathrm{r}_{2}^{2}}}{\mathrm{r}_{1}} \tan \left(\frac{\mathrm{r}_{1} \theta+\mathrm{r}_{2}}{\sqrt{\mathrm{r}_{1} \mathrm{r}_{3}-\mathrm{r}_{2}^{2}}}\right)
$$

This formula is obviously of the form given in equation (4). If now the parameter $\theta$ is taken as a linear function of the Barbilian phase, the equation (11) can be interpreted as the mean stress on a certain plane. This very plane is not so important by itself for the moment, and for our present purposes can be identified with a cross-sectional plane of the experimental specimen. Then the numbers $r_{1}, r_{2}, r_{3}$ or their counterparts in experimental records:

$$
\begin{aligned}
& \mathrm{x}=\mathrm{A}+\mathrm{B} \tan (\alpha \theta+\beta) \\
& \mathrm{A} \equiv-\frac{\mathrm{r}_{2}}{\mathrm{r}_{1}} ; \quad \mathrm{B} \equiv \frac{\sqrt{\mathrm{r}_{1} \mathrm{r}_{3}-\mathrm{r}_{2}^{2}}}{\mathrm{r}_{1}} ; \quad \alpha \equiv \frac{\mathrm{r}_{1}}{\sqrt{\mathrm{r}_{1} \mathrm{r}_{3}-\mathrm{r}_{2}^{2}}} ; \quad \beta \equiv \frac{\mathrm{r}_{2}}{\sqrt{\mathrm{r}_{1} \mathrm{r}_{3}-\mathrm{r}_{2}^{2}}}
\end{aligned}
$$

are certainly related to the internal 'molecular' properties of the specimen. In the specific case of rubbers these properties are those of the macromolecular chains and filler dispersions. In the case of nuclear matter, the 'molecules' may be replaces by quarks, partons, mesons, baryons, etc.

\section{Statistics of Intermolecular Forces}

The above considerations leave one important subject uncovered: the actual relationship between the parameter $\theta$ of the family of quadratic variance distribution functions and the experimental recording. All we can say is what already has been said before, namely that, in the specific problem of deformation, the 
engineering experience to date indicates that the phase $\phi$ of representation of the stresses is in direct connection with the progress of deformation. This fact is reinforced through the close representation of experimental data for uniaxial tension (or compression) by equation (4) with the phase depending linearly on the recorded deformation (see Figs 1 and 2). If, in a general situation, the deformation is experimentally quantified by the experimental stretch $\lambda$, then a relationship between $\theta$ and $\lambda$ is to be expected, for $\theta$ is, in general, a function of the experimental recording. We are now in no position of finding directly this relationship, but there is an indirect way to it, namely the agreement with experimental data.

According to our philosophy, the experimental stress must be a mean. Then how do we account for a mean that is rational function of stretch? In order to answer this question, notice that everything in the general exponential statistics depends on the a priori measure $\mu(d \xi)$ we use for the characterization of the ideal continuum approximating the real body. Thus we must find the a priori measures that best suit our experimental needs. These measures may not be always positive, but the fact remains that regardless of this feature they must always give results in finite terms. In order to exemplify this point we choose some polynomial measures as given in Table I. The table offers the normalization factors as functions of the parameter $\theta$ for different a priori polynomial measures of the real line. By the particularly simple transformation

$$
\theta=\lambda^{-1}
$$

we can bring these normalization factors to experimental terms, and see if we can get a real situation out of this. To make a long story short, we take the situation represented by the last row of the Table I in terms of the physical parameter $\lambda$ :

$$
\mathrm{dF}_{\mu}(\mathrm{x})=\frac{1}{\lambda\left(2 a \lambda^{2}+2 b \lambda+c\right)} \mathrm{e}^{-\frac{\mathrm{x}}{\lambda}}\left(a x^{2}+2 b x+c\right) d x
$$

For this family of distributions the mean is

$$
\bar{x}=\lambda \frac{6 a \lambda^{2}+4 b \lambda+c}{2 a \lambda^{2}+2 b \lambda+c}
$$

Now, the relation between experimental recording and the parameter of the distribution may not be as simple as that from equation (18), but the fact is that the mean over such ensemble can preserve its simple functional form (18) in very large conditions, perhaps with more parameters though. Such a mean can then be further cast into the form of a sum of linear fractional functions of stretch:

$$
\overline{\mathrm{x}}=\mathrm{A}+\mathrm{B} \lambda+\frac{\mathrm{C}}{\lambda+\alpha}+\frac{\mathrm{D}}{\lambda+\beta}
$$

which is more suitable for both practical, and especially theoretical purposes.

The experimental force must be proportional with the quantity (18) (or (19), does not really matter), which thus replaces the equation (4) from the ideal uniaxial and even biaxial or planar cases. Notice that what is really changing here is not the nature of the statistic to be considered - it is always given by a quadratic variance distribution function - but the experimental measure of the stage of deformation characterizing the ensembles of equilibrium intermolecular forces inside rubber. Also, notice that the stretch in the present case replaces the temperature from the classical case of ideal gas, as a parameter of 
the equilibrium distribution. In that case the statistic would refer to kinetic energy, here the statistic refers to forces and the parameter charactrizes the stage of deformation. Fig. 3 shows the quality of representing experimental data by function (19).

\section{An Energy Function}

In view of equation (19), the nominal stress $\sigma$ will then be arranged in a particularly convenient form as

$$
\sigma(\lambda)=(\lambda-1)\left(\alpha+\frac{\beta}{\lambda-1-a}+\frac{\gamma}{\lambda-1-b}\right)
$$

with $\alpha, \beta, \gamma, \mathrm{a}, \mathrm{b}$ - some constants coming from experiment. Here we chose for reference the undeformed state of the specimen. The advantage of using equation (20) for the nominal stress becomes more obvious if we integrate it in order to obtain the energy function for the model. The result is

$$
\mathrm{U}(\lambda)=\frac{\alpha}{2}(\lambda-1)^{2}+(\beta+\gamma)(\lambda-1)+\beta \mathrm{a} \ln \left|\frac{\lambda-1}{\mathrm{a}}-1\right|+\gamma \mathrm{b} \ln \left|\frac{\lambda-1}{\mathrm{~b}}-1\right|
$$

Now, equation (21) can be advantageously used if we accept further assumptions, helping to go over from the uniaxial case to the general three-dimensional case, namely that the stretch is indeed a tensor, and that what we measure in experiment is just one or more of its eigenvalues. Assuming then isotropy, to the effect that equation (20) is the same no matter of the eigenvalue of the stretch tensor, and the Valanis-Landel hypothesis, stating that the total energy function is the sum of the three components of the energy along the principal directions of stretch tensor (Valanis, Landel, 1967), we finally get

$$
\begin{array}{r}
\mathrm{U}=\frac{\alpha}{2}\left(\mathrm{I}_{1}-2 \mathrm{~K}_{1}+3\right)+(\beta+\gamma)\left(\mathrm{K}_{1}-3\right) \\
+\beta \mathrm{a} \ln \left|\frac{\mathrm{K}_{3}-(\mathrm{a}+1) \mathrm{K}_{2}+(\mathrm{a}+1)^{2} \mathrm{~K}_{1}-(\mathrm{a}+1)^{3}}{\mathrm{a}^{3}}-1\right| \\
+\gamma \mathrm{b} \ln \left|\frac{\mathrm{K}_{3}-(\mathrm{b}+1) \mathrm{K}_{2}+(\mathrm{b}+1)^{2} \mathrm{~K}_{1}-(\mathrm{b}+1)^{3}}{\mathrm{~b}^{3}}-1\right|
\end{array}
$$

Here we have used the notations

$$
\mathrm{K}_{1}=\lambda_{1}+\lambda_{2}+\lambda_{3} ; \quad \mathrm{K}_{2}=\lambda_{2} \lambda_{3}+\lambda_{3} \lambda_{1}+\lambda_{1} \lambda_{2} ; \quad \mathrm{K}_{1}=\lambda_{1} \lambda_{2} \lambda_{3}
$$

where $\lambda_{1,2,3}$ are the three eigen-stretches. Notice that this energy is not a pure first and second invariant theory, of the kind used by Nicholas Manton in his geometrization, and this fact algebraically complicates the matter. However, the complication is no more than algebraic and it can be easily overcome. Notice also that we have here both a generalization of the so-called neo-Hookean model, actually of a so-called Varga type constitutive model (Varga, 1966). This result is particularly encouraging in the general endeavor of constitutive modeling. However, it is especially encouraging in the characterization of the nuclear matter, by extending what we would like to call a 'Manton program', for which the work of Manton on the geometrization of skyrmions (Manton, 1987) is the inception point. 


\section{Application to Nuclear Matter}

Nuclear matter is first of all, obviously, matter. Here the internal forces between its structural constituents are critical ingredients in understanding and explaining its structure, and that even in a much larger extent than in any classical instance of matter. These forces are especially used in explaining the idea of confinement, and the concept of deconfinement coming naturally with it (Satz, 1986, 1994, 2000). The constitution of nuclear matter is here, like everywhere in physics, assumed a priori and, because of that, the forces are usually described by a potential whose structure is adjusted by phenomenological arguments. Typical among others, remains the idea that the confinement refers to quarks, bound to their hadrons for instance, and that it is described by a potential which, in a first approximation is linear in the quark separation 'r' (Satz, 2000). This would explain the confinement of quarks within the structure of hadrons, inasmuch as its deconfinement requires an infinite energy in order to be carried on. In broad lines, however, the potential is an unknown, and one usually resorts upon computer experiments based, for instance, on lattice calculations in order to get some insight on its functional form (Alkofer, Greensite, 2007).

The quarks, as well as partons, are remnants of the classical view of a ultimate structure of matter in the specific field of nuclear matter. Fact is that, ever since the discovery of mesons, the idea occurred that the baryons are not stable particles in the structure of nucleus, but are in a continuous change within that structure. This continuous change was first explained by exchange of mesons. It is nowhere recognized in theoretical physics that the concept of particle potential in the structure of nuclear matter might be not only too poor for the representation, but also totally inappropriate. For, the nuclear matter, either static or dynamic is first of all matter. All one can say about it, in view of the manifest 'dynamism' of nuclear matter, is that the force centers, necessary in the mathematical description by potential, are accidental and can vary randomly in space and time. Therefore these force centers should be described by a stochastic process in some kind of collective coordinates.

More to the point, the potential, as classically understood, is hardly a reasonable description of the confined matter, as the very idea of confinement involves a dynamical grouping of the accidental centers of force in the matter. This dynamics is dictated by external reasons, which can be generically gathered under the label 'deformation'. In reality therefore, the nuclear matter should be described by arguments closer to the theory of deformation rather than by the theory of point particles connected by central forces. This fact makes the Manton's idea a central point, the inception of a program actually - the 'Manton program', as we called it before. In this respect, the classical experimental loading curves are the best candidate in describing the confinement, for they reflect the internal forces of the matter. We just have to assume that the confinement is independent of the behavior of external loading force: this one may be even a sudden impact force. The first thing the loading curves show is that one does not have to go to infinity in order to get an infinite force of 'deconfinement'. The infinite force is achieved at a finite distance, in the places where a rational map like that from equation (21) has a vertical asymptote. The deconfinement is really achieved by some other means than ripping the matter, as indeed the phenomenology indicates. The core device of this approach is indeed the rational map which turns out to be a correspondence between the mean of the ensemble representing the equilibrium of matter, and the experimental parameter(s) of the family of distributions representing this ensemble.

A few references in the analogy of nuclear matter with some classical concepts are worth bringing into discussion from this point of view. The first concept historically entering the analogy was that of the ideal gas. The parameter of the ensemble classically describing it at thermodynamic equilibrium is the (absolute) 
temperature. Any forces between its structural constituents - the molecules - are eliminated by reasons of sufficiency (Jeans, 1954): the temperature is considered a sufficient statistic for the kinetic energy of the molecules. The first alarm signal regarding this model occurred when the temperature was taken as defining also the thermal equilibrium for the blackbody radiation. There, the first change in physical reasoning has appeared, for the ensemble describing the equilibrium of this physical system is of the quadratic variance distribution function type, described by equation (12) above, whereby $\theta$ is the reciprocal of temperature and ' $x$ ' is the spectral energy density of radiation (Planck, 1900). The harmonic oscillator was thus established as the fundamental unit of any model in the structure of matter. But then it was discovered (Compton, 1915) that, from this point of view, the energy of a solid has to have a constant term added to it, which cannot be accounted for by extending the classical theory of ideal gas. Indeed, such a term can only be explained by long distance forces between the molecules of the ideal gas, and these forces were among those eliminated by the classical equation of state of an ideal gas in the first place. They correspond to a logarithmic potential, which according to some classical arguments can be accepted only for a solid, or corresponds cosmologically to inertia forces (Sciama, 1969). Well, it seems that the forces eliminated a century ago from the realm of gases will continue to haunt us, for the logarithmic potential comes again to light in the case of nuclear matter, in a pure Chern-Simons gauge argument regarding the construction of the confining potential (Gaete, 2003, 2005). There is still much to learn from the classical gas after all!

No wonder then, a classical ideal gas model for nuclear matter (Fermi, 1950) could not give full satisfaction: the ideal gas structure of the matter is not itself satisfactorily described from theoretical point of view. On the other hand, for a solid, like for the blackbody radiation, the temperature does not reflect a sufficient statistic anymore. But, while in the case of the blackbody radiation we would have the frequency to support the complementary experimental information, for a solid this is no more the case, and so much the more for the nuclear matter. We are facing here the problem of choice of a parameter for the theoretical distributions characterizing an equilibrium state of the matter. The Manton's geometrization then points to the fact that, as long as that equilibrium state refers to the deformation of (nuclear) matter, the stretches are the parameters of choice. As shown above, in a general definition, they are related to the means of the ensembles of equilibrium by rational maps. They lead to a general form of the confining potential, to be unveiled, in a particular scaling case, in the next section.

\section{A Typical Confining Potential}

With the previous considerations the door opens for still other side of the analogy that gets into the theoretical description of the nuclear matter. This time the analogy refers to the Newtonian forces proper. Namely, if the forces of confinement in matter are described by a potential, then the best candidate for such a potential should be, in general, the function from equation (22). Manton's work just opens therefore the way of an exquisite phenomenological theory involving the very logarithmic potential, which appears presently in the last two terms of the potential from equation (22). One could say from the outset that, inasmuch as that equation is surely approximate by the way we got it, the logarithmic potential might be also the expression of an approximation. In order to see what kind of approximation it is, one only needs some natural assumptions on the structure of nuclear matter, among which the existence of partons as centers of forces and of quarks as centers of the 'resultants' of these forces, come naturally handy. 
However, one can get some information just by considering the potential as it is, with no structural assumptions yet.

We describe a model of nuclear matter as close as possible to the blackbody radiation, not the ideal gas. This involves a particular form of equation (22) for which the three stretches are equal. This particular situation is termed by Derrick 'uniform scaling' (Derrick, 1964; Manton, 2009). Indeed, in this case one can prove that the essential physical condition for the theoretical description of blackbody radiation is satisfied (Mazilu, 2010a,b; Mazilu, Agop, 2012). That condition comes down to Wien's displacement law, in its general expression which serves as fundamental criterion in establishing the functional form of the spectral density of radiation as a function of frequency and temperature. In the like case, equation (22) produces a potential of the form given in equation (21) up to an unimportant factor 3:

$$
\begin{array}{r}
\mathrm{U}=\alpha\left(\mathrm{r} / \mathrm{r}_{0}-1\right)^{2} / 2+(\beta+\gamma)\left(\mathrm{r} / \mathrm{r}_{0}-1\right) \\
+\beta \mathrm{a} \ln \left|\left(\mathrm{r} / \mathrm{r}_{0}-1\right) / \mathrm{a}-1\right|+\gamma \mathrm{b} \ln \left|\left(\mathrm{r} / \mathrm{r}_{0}-1\right) / \mathrm{b}-1\right|
\end{array}
$$

where $r$ is the space separation between the constituent points of the matter (the instantaneous centers of force), and $r_{0}$ is its reference value. This gives a central force with the magnitude depending only on the distance between nuclear particles, considered as centers of force, in the form

$$
\mathrm{F}(\mathrm{r})=\left(\mathrm{r} / \mathrm{r}_{0}-1\right) \cdot\left(\alpha+\frac{\beta}{\mathrm{r} / \mathrm{r}_{0}-1-\mathrm{a}}+\frac{\gamma}{\mathrm{r} / \mathrm{r}_{0}-1-\mathrm{b}}\right)
$$

In the limit of high separation, the linear term prevails, so the confining force depends there linearly on the separation between the constituents of the nuclear matter: it is not a constant. Depending on a interplay of the parameters, the potential from equation (24) may reduce, for instance to the known "Cornell potential", containing the electric forces and the constant forces. But here we would like to point out something else regarding this potential.

\section{Conclusions and Outlook}

The calculations of the static potential are usually done by constructing the partition function over a structural ensemble. The structure here refers to matter, and the partition function requires the parameter of the distribution to be used in calculation of the partition function. Currently, this parameter is the remnant of the temperature from the classical theory of the ideal gas. Useless to say, this parameter is not sufficient from many points of view. First of all, it does not reflect a sufficient statistic, but only in the original use, viz. only for the classical ideal gas. Secondly, in the case of nucleus the space extension of particles becomes important, and the statistics should reflect this in a natural way.

Starting from the thermal radiation, and continuing on through the theory of solids, one feels indeed necessary to introduce some other parameters. Now, if in the description of nuclear matter one has to use pertinent analogies, then the standards of these analogies should themselves be properly described from a physical point of view. This is far from being currently the case. One of the standards of analogy for the nuclear matter is the solid state of the matter. It is not itself a properly described concept, but offers however a way out of difficulty through the practice of deformation, and its associated theories, as described above. This practice is able to give us a proper reference static potential, by providing a statistic whose parameter is different from temperature. This parameter reflects the essence of extended particles 
suspected to enter the structure of nuclear matter. It is the Barbilian phase, measuring the capability of extended matter to "deter", so to speak, the action of external forces from the direction sought for of this action. Therefore a statistical ensemble referring to nuclear matter should have a distribution function characterized by two parameters: the temperature and the Barbilian phase of 'transverse motion'.

This philosophy produced here a potential having some basic properties required in different structural theories of the nuclear matter. Chief among the traits of this potential is that it contains naturally a logarithmic term, which we presume to be a remnant from the classical theory of ideal gas. Going into specifics, in Fig. 4 the static potential (24) is represented, for the parameters found by curve fit of the data from Fig. 1. The origin of potential was conveniently chosen to be zero, in order to avoid complications which, for the moment, would only side-track our conclusions. The behavior shows another basic property of the potential: the infinite gradual increase with the separation, as indeed one feels it should be the case. However, there are reasons to believe that this potential saturates somewhere, in the sense that the force becomes zero, and that this event is physically meaningful, for instance in the phase transitions of the matter. This would mean that the expression we gave for the potential has to be only approximate, as it was intended, indeed. But the challenge now is to find just what approximation is that. The following considerations may help in the quest.

From a structural point of view, a pure gauge theory could probably be able to account in full, so to speak, for this general behavior, by reestablishing the long distance logarithmic potential from the case of ideal gas in its natural rights. In any case, it is able to account approximately for such a potential (see Gaete, 2003, 2005). Related to this idea, an observation is perhaps relevant here, that can guide our future systematic approaches of the problem. The approach of Patricio Gaete, in the works cited above, produces a potential of the form $\mathrm{K}_{0}\left(\mathrm{r} / \mathrm{r}_{0}\right)$, where $\mathrm{K}_{0}$ is the modified Bessel function of second kind. Then the general relations (Gradshteyn, Ryzhik, 2007, Ex. 8.447.1 \& 3):

$$
\mathrm{K}_{0}(\mathrm{z})=-\ln \frac{\mathrm{z}}{2} \cdot \mathrm{I}_{0}(\mathrm{z})+\sum_{\mathrm{k}=0}^{\infty}\left(\frac{\mathrm{z}^{\mathrm{k}}}{2 \mathrm{k} !}\right)^{2} \psi(\mathrm{k}+1) ; \quad \mathrm{I}_{0}(\mathrm{z})=\sum_{\mathrm{k}=0}^{\infty}\left(\frac{\mathrm{z}^{\mathrm{k}}}{2 \mathrm{k} !}\right)^{2}
$$

where $\mathrm{I}_{0}$ is the modified Bessel function of the first kind, and $\psi$ is Euler's psi-function, show that in the approximation of small separation the logarithmic term dominates, hence the logarithmic potential is effective there (Gaete, 2003). In other words the logarithmic potential itself is just an aproximate numerical case, depending on the separation distance between the centers of force inside the nuclear matter.

One can see, from the shape of potential function Fig. 4, that the behavior of the potential is dictated almost exclusively by the polynomial terms from its expression. Therefore, in this approximation, we need to concentrate on this part, and not on the logarithm, in order to find a closed-form expression that would represent the approximate potential. Fig. 5 shows that the approximate potential given by equation (24) accepts indeed a closed functional form, involving ab initio the modified Bessel function of the first kind:

$$
\mathrm{U}(\mathrm{r})=\mathrm{Ae}^{\mathrm{B} \cdot\left(\mathrm{r} / \mathrm{r}_{0}-1\right)} \mathrm{I}_{0}\left[\mathrm{C} \cdot\left(\mathrm{r} / \mathrm{r}_{0}-1\right)\right]
$$

If this is the case, then the true potential should have this form in a wide enough range of separations, not the logarithmic form, which should prevail at larger separations. For the form (27) there are theoretical reasons related to the statistics on Wilson loops in two dimensions, and these very reasons can be applied for the general form of the confining potential. This issue will be treated in a future work, but we can 
indicate even by now the source of the term from equation (27), and thus the basis of that future work, for it contains some interesting structural considerations.

It is quite obvious that, in conducting the considerations of the present work, the a priori measure $\mu(\lambda)$ of the real axis is instrumental. It is here the equivalent of the a priori lattices from the usual approach, and needs to be ascertained before we start any speculations. No doubt, the expression from equation (24) is approximate by the very fact that we used just guesses for this a priori measure. For instance the curve from Fig. 1 is well represented by a sum of two or three linear fractional functions, covering one or another of its portions. This circumstance led to the idea of rational function for the curve fit in the first place. Now, the measure $\mu$ depends exclusively on the ensemble serving to perform the statistical calculations, and it is here where we need to concentrate our effort.

The Barbilian phase itself, reflects another sufficient statistic on such an ensemble, just like the temperature for the classical ideal gas, and this statistic is related to frequency. Indeed, in the limit where we consider the thermal radiation, or indeed a solid, as an ensemble of oscillators, each one of the elements of this ensemble, having a given frequency, is coordinated by three parameters, representing a complex amplitude and a real phase. This real phase is, again, the Barbilian phase. The frequency per se is therefore a sufficient statistic, which can be produced as instantaneous frequency (the mean of time derivative of phase), or a function of frequency, on such an ensemble. Going further, the Barbilian phase should be therefore naturally related to color, once it is related to frequency. It is here the point where group theory arguments produce an a priori measure for color, and therefore a propagator of color on such an ensemble. This is a function that, in the particular case of uniform scaling, reduces to that from equation (27).

Therefore the color of structural members of the ensemble representing a nucleus is the one responsible for such a behavior as that reflected in the potential (27). Indeed one can prove, based on classical arguments, that the color is a parameter characterizing in a special way the nuclear extended particles, just as the static charge does it for their classical point counterparts. One can say that within nuclear matter the forces are indeed not independent of one another. This seems just a vague statement, repeating the one that actually started our argument in the first place. In fact, however, it seems that the original concept of current quantum chromodynamics involving the lines of force can be improved by specifying the way in which the forces depend on each other. Specifically, the forces due to color actually act upon the flux of electric or magnetic lines of force. This, or one like this, is the only sound physical conclusion of a classical theory so formulated. As we said, we reserve a future occasion for the details of such a theory. 


\section{References}

Alkofer, R., Greensite, J. (2007): Quark Confinement: the Hard Problem of Hadron Physics, Journal of Physics G: Nuclear and Particle Physics, Vol. 34, pp. S3-S21

Bali, G. S. (2001): QCD Forces and Heavy Quark Bound States, Physics Reports, Vol. 343, pp. 1-136

Charalampidis, E. G., Ioannidou, T. A., Manton, N. S. (2011): Skyrmions, Rational Maps \& Scaling Identities, Journal of Mathematical Physics, Vol. 52, 033509; arxiv:hep-th/1012.3778v1

Compton, A. H. (1915): The Variation of the Specific Heat of Solids with Temperature, Physical Review, Vol. 6, pp. 377-389

Derrick, G. H. (1964): Comments on Nonlinear Wave Equations as Models for Elementary Particles, Journal of Mathematical Physics, Vol. 5, pp. 1252-1254

Enz, U. (1963): Discrete Mass, Elementary Length, and a Topological Invariant, as a Consequence of a Relativistic Invariant Variational Principle, Physical Review, Vol. 131, pp. 1392 - 1394

Enz, U. (1964): Die Dynamik der Blochschen Wand, Helvetica Physica Acta, Vol. 37, pp. 245 - 251

Enz, U. (2006): Rotating Hopf Kinks: a Realistic Particle Model, Physica D, Vol. 223, pp. 1 - 6

Fermi, E. (1950): High Eenergy Nuclear Events, Progress of Theoretical Physics (Japan), Vol. 5, pp. 570 583

Gaete, P. (2003): Static Potential and a New Generalized Connection in Three Dimensions, arXiv:hepth/0305025v1

Gaete, P. (2005): On External Backgrounds and Linear Potential in Three Dimensions, arXiv:hepth/0501136v1

Gradshteyn, I. S., Ryzhik, I. M. (2007): Table of Integrals, Series and Products, Seventh Edition, A. Jeffrey \& D. Zwillinger Editors, Academic Press-Elsevier, Inc.

Hobart, R. H. (1963): On the Instability of a Class of Unitary Models, Proceedings of the Physical Society of London, Vol. 82, pp. 201-203

Jeans, J. H. (1954): The Dynamical Theory of Gases, Dover Publications, New York

Manton, N. S. (1987): Geometry of Skyrmions, Communications in Mathematical Physics, Vol. 111, pp. $469-478$

Manton, N. S. (2009): Scaling Identities for Solitons Beyond Derrick's Theorem, Journal of Mathematical Physics, Vol. 50, 032901

Manton, N. S. (2012): Classical Skyrmions - Static Solutions and Dynamics, Mathematical Methods in Applied Sciences, Vol. 35, pp. 1188-1204; arxiv:hep-th/1106.1298v1

Mazilu, N. (2006): Geometrical Theories Around Cubic Equation, www.protoquant.com

Mazilu, N. (2010a): A Case Against the First Quantization, viXra:quantum physics/1009.0005/

Mazilu, N. (2010b): Black-Body Radiation Once More, Bulletin of the Polytehnic Institute of Iaşi, Vol. 56, pp. 69-97

Mazilu, N., Agop, M. (2012): Skyrmions - a Great Finishing Touch to Classical Newtonian Philosophy, Nova Publishers, New York

Mooney, M. (1940): A Theory of Large Elastic Deformation, Journal of Applied Physics, Vol. 11, pp. 582592

Planck, M (1900): Planck's Original Papers in Quantum Physics, translated by D. Ter-Haar and S. G. Brush, and Annotated by H. Kangro, Wiley \& Sons, New York 1972 
Rivlin, R. S. (1948): Large Elastic Deformations of Isotrpic Materials, Philosophical Transactions of the Royal Society of London, Vol. A241, pp. 379-397

Satz, H. (1986): Quark Deconfinement and High Energy Nuclear Collisions; Preprint BNL 38530

Satz, H. (1994): Deconfinement: Concept, Theory, Test; Preprint CERN-TH 7410/94

Satz, H. (2000): Colour Deconfinement in Nuclear Collisions, Reports on Progress in Physics, Vol. 63, pp. 1511-1574

Satz, H. (2011): The Quark-Gluon Plasma - A Short Introduction, Nuclear Physics A, Vol. 862-863, pp. 4-12

Sciama, D. W. (1969): The Physical Foundations of General Relativity, Doubleday, New York

Valanis, K. C., Landel, R. F (1967): The Strain Energy Function of a Hyperelastic Material in Terms of the Extension Ratios, Journal of Applied Physics, Vol. 38, pp. 2997 - 3002

Varga, O. H (1966): Stress-Strain Behaviour of Elastic Materials, Interscience, New York 
Table I

A List of Polynomial Measures and Corresponding Normalization Factors

\begin{tabular}{ll}
\hline A Priori Measure & Normalization Factor \\
\hline$\mu(d \xi)=d \xi$ & $N_{\mu}(\theta)=\frac{1}{\theta}$ \\
\hline$\mu(d \xi)=\xi d \xi$ & $N_{\mu}(\theta)=\frac{1}{\theta^{2}}$ \\
\hline$\mu(d \xi)=\xi^{2} d \xi$ & $N_{\mu}(\theta)=\frac{2}{\theta^{3}}$ \\
\hline$\mu(d \xi)=\xi^{3} d \xi$ & $N_{\mu}(\theta)=\frac{6}{\theta^{4}}$ \\
\hline$\mu(d \xi)=\xi^{4} d \xi$ & $N_{\mu}(\theta)=\frac{24}{\theta^{5}}$ \\
\hline$\mu(d \xi)=(a \xi+b) d \xi$ & $N_{\mu}(\theta)=\frac{a+b \theta}{\theta^{2}}$ \\
\hline$\mu(d \xi)=\left(a \xi^{2}+2 b \xi+c\right) d \xi$ & $N_{\mu}(\theta)=\frac{c \theta^{2}+2 b \theta+2 a}{\theta^{3}}$ \\
\hline
\end{tabular}




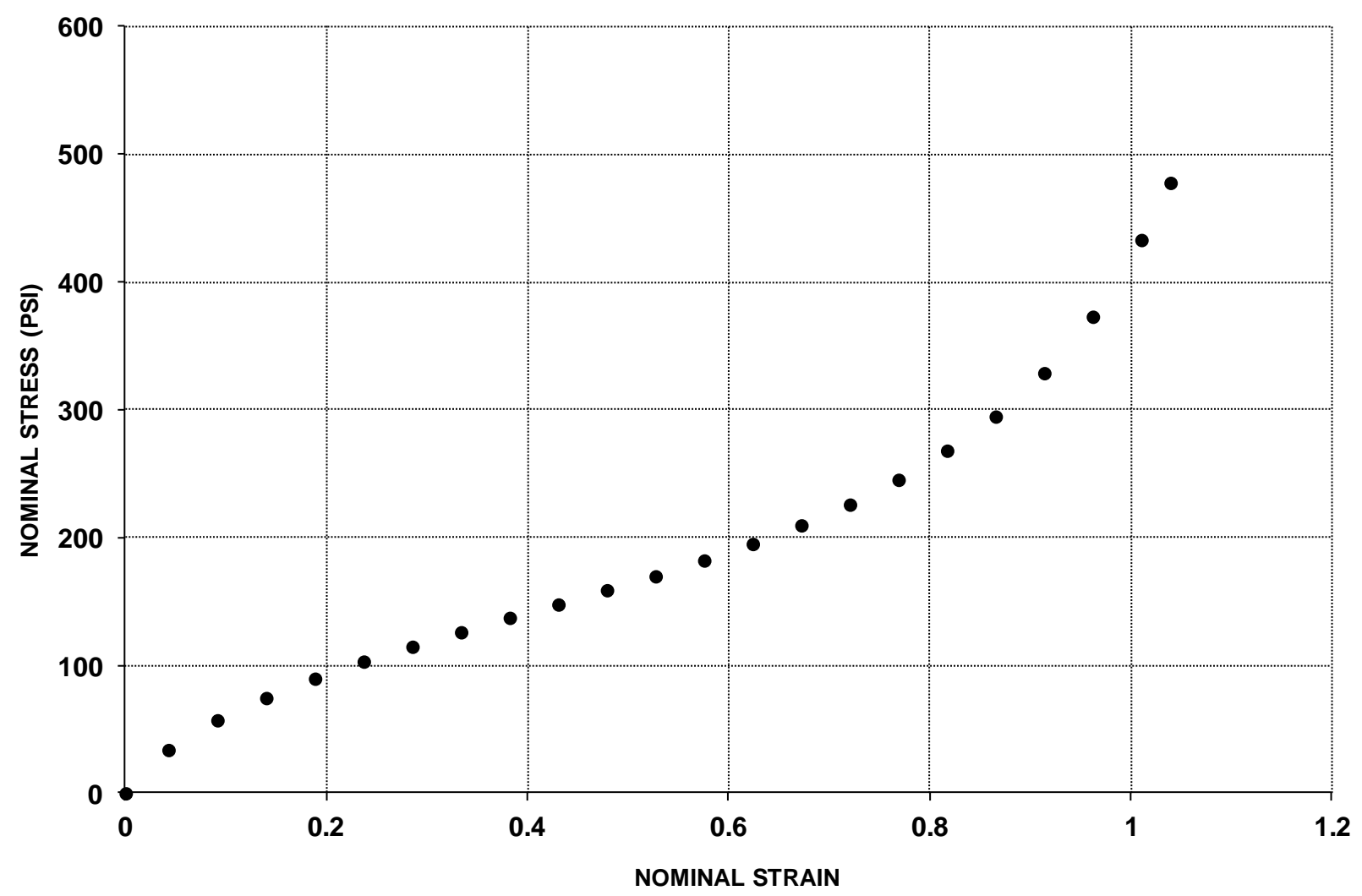

Fig. 1. The data points of a typical loading curve of cured rubber, extended a little over 100\% elongation. Imperial units are used for the stress, and therefore for the density of energy. 


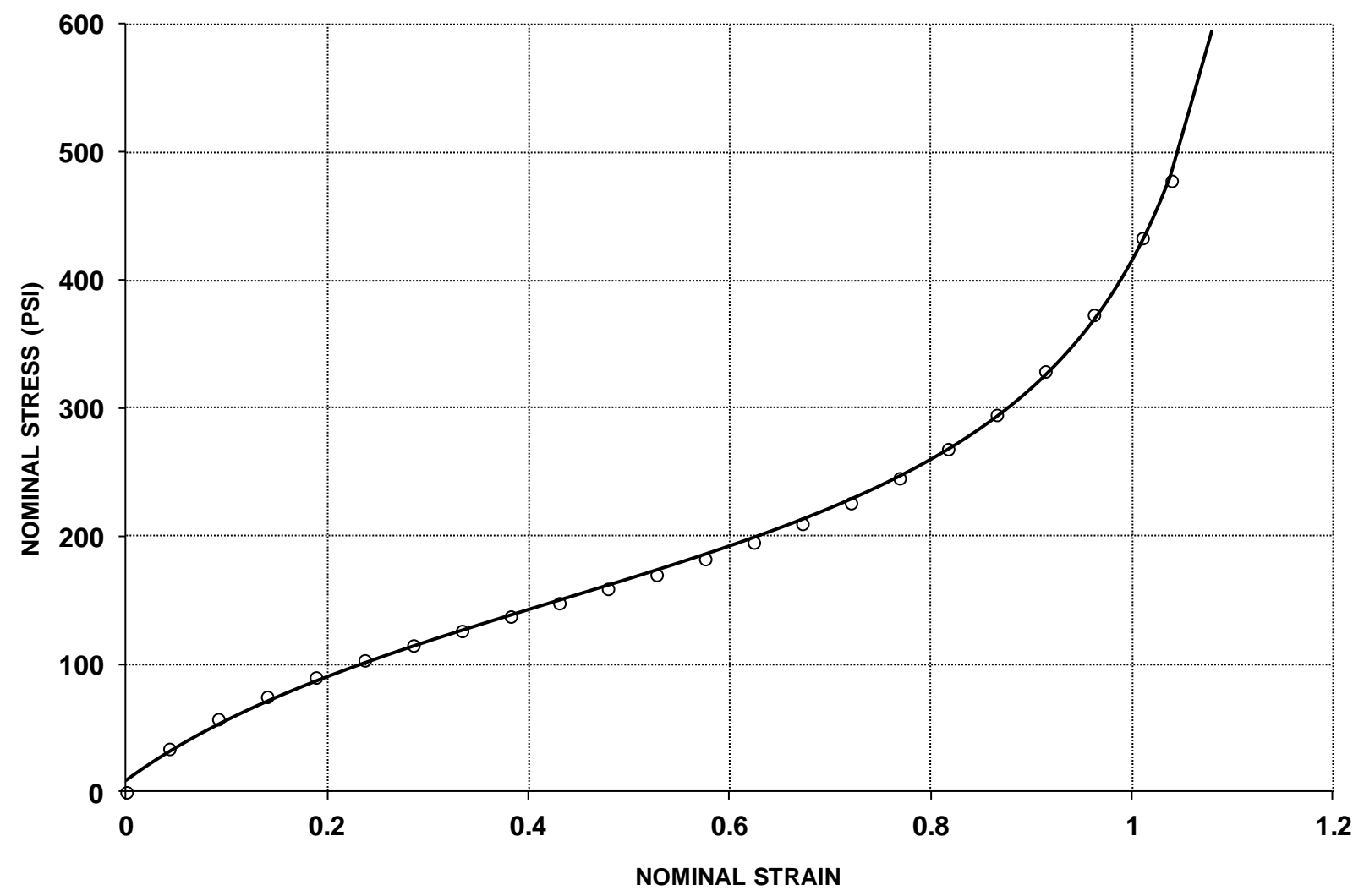

Fig. 2. The 'tangent fit' (full line) of the data (circles) from Fig. 1. The argument of the tangent function is here a linear function of the stretch, even though the data is represented as a function of nominal strain. One can see clearly a little misfit in the region of origin of strains. This may be due to the fact that we chose a linear function for the argument of the tangent function (see text). 


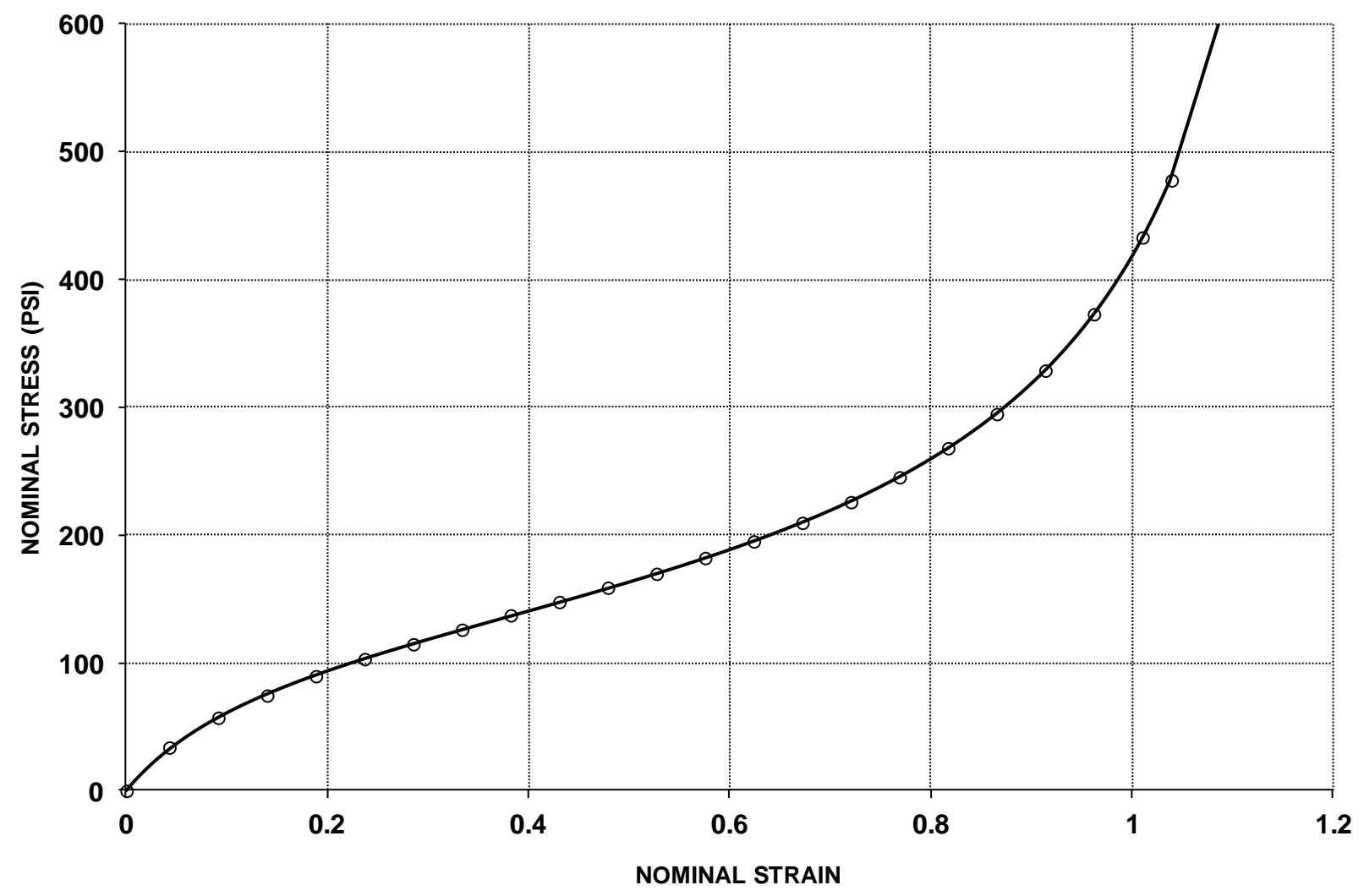

Fig. 3. The 'rational fit' (full line) of the data (circles) from Fig. 1. The argument of the rational function is here the nominal strain. One can see that the curve-fit is better than that from Fig. 2 (see text). 


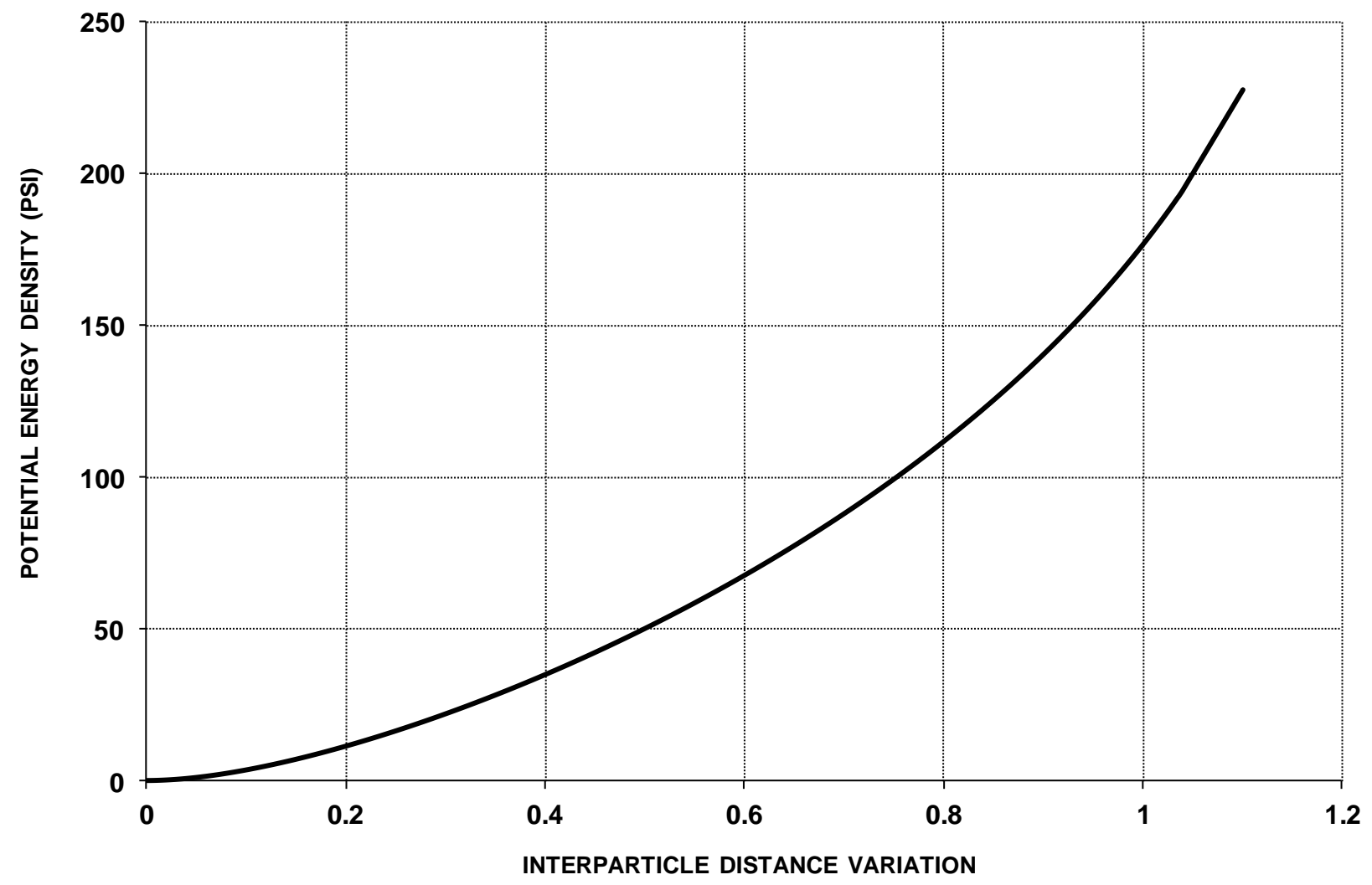

Fig. 4. The potential energy density based on the 'rational fit' from Fig. 3 of the data from Fig. 1. The 'quadratic function' behavior, specific for the case of solids or plasmas is clearly dominating. 


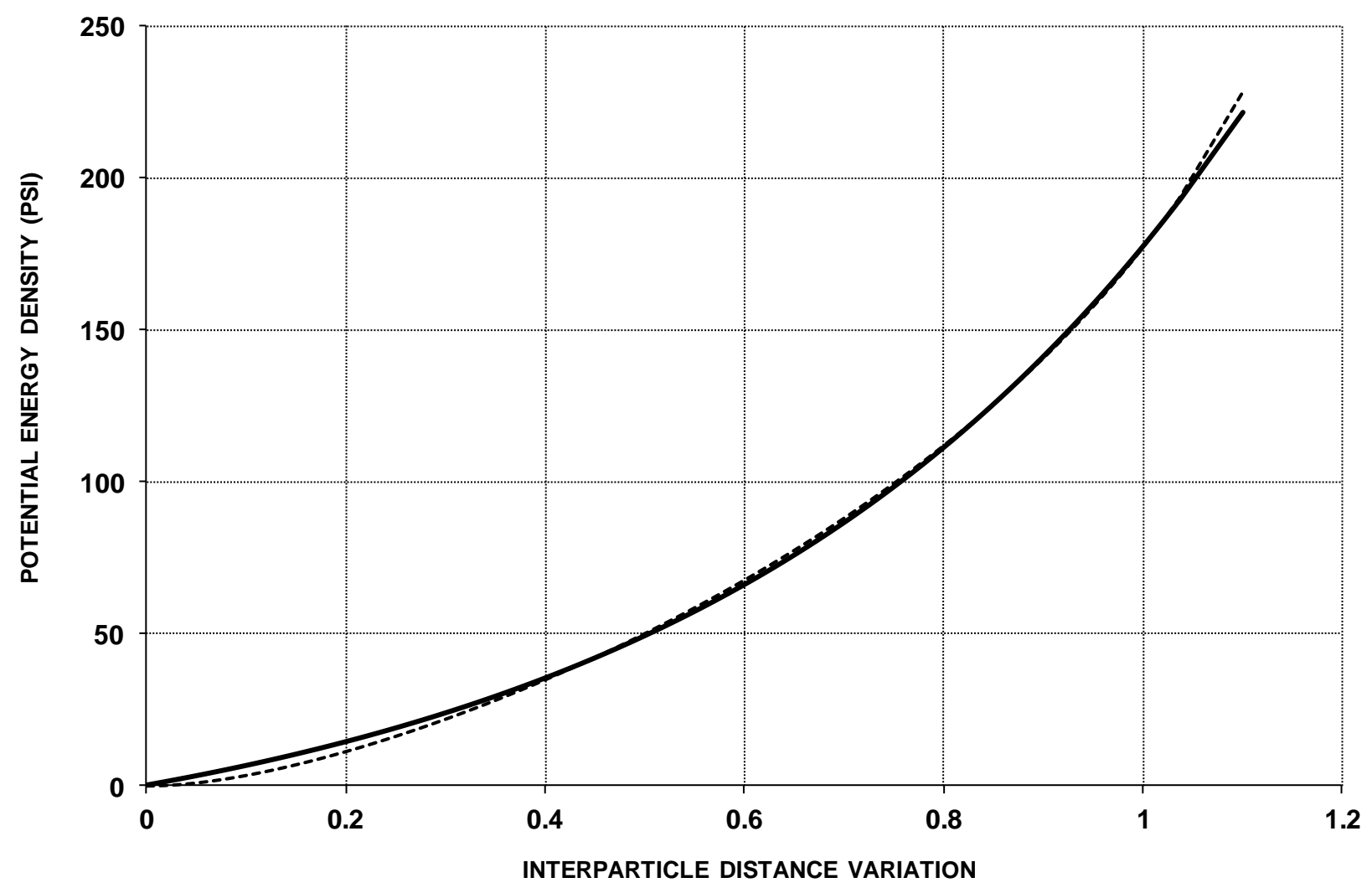

Fig. 5. The potential energy density based on the 'modified Bessel function of first kind fit' (full line; see text) of the potential from Fig. 4 (dashed line). Even though the two curves do not coincide, they are nevertheless very close. Here we assume that the full line is the true approximate physical result. 